tượng thiếu hut kiến thức chủ yếu về nội dung điều trị biến chứng của bệnh như: chăm sóc bàn chân, kiểm soát hạ đường huyết. Điểm trung bình (thang điểm 4) của các đối tượng đạt được là $2,48 \pm 0,90$. Tỷ lê đối tượng đạt mức kiến thức cao ( $>2$ điểm) chiếm $67,9 \%$.

Lời cảm ơn: Nghiên cứu này được Bộ Ngoại giao Đan Mạch tài trơ qua dự án "Sống chung với bênh man tính: Hổ trơ khồng chính thức cho quản lý bệnh đái tháo đường ở Việt Nam" (số 17-M09-KÜ). Chúng tôi rất cảm ơn các cơ quan y tế tuyến tỉnh, huyện, xã tại Thái Bình đã giúp đõ chúng tôi thu thập thông tin cho cuộc khảo sát.

\section{TÀI LIÊU THAM KHẢO}

1. World Health Organization (WHO). Global Report on Diabetes 2016. https:// apps.who.int/iris/handle/10665/204871

2. McInnes, AD. Diabetic foot disease in the United Kingdom: about time to put feet first. J Foot Ankle Res 5, 26 (2012). https://doi.org/10.1186/17571146-5-26

3. Whiting DR, Guariguata $L$, Weil $C$, et al. IDF diabetes atlas: global estimates of the prevalence of diabetes for 2011 and 2030. Diabetes Res Clin Pract. 2011 Dec; 94(3):311-21.

4. International Diabetes Federation (IDF). IDF
Diabetes Atlas, http://fmdiabetes.org/wpcontent/uploads/2018/03/IDF-2017.pdf

5. Nguyen BN, Zhou LL and Waqas AD. Diabetes: What Challenges Lie Ahead for Vietnam? Ann Glob Health. 2020; 86(1): 1. Published online 2020 Jan 2. doi: $10.5334 /$ aogh. 2526

6. Rosland AM, Piette JD, Choi $\mathrm{H}$, et al. Family and friend participation in primary care visits of patients with diabetes or heart failure: patient and physician determinants and experiences. Med. Care. 2011; 49: 37-45.

7. Messenger G, Taha N, Sabau S, et al. Is there a role for informal caregivers in the management of diabetic foot ulcers? A narrative review. Diabetes Ther. 2019; 10(6): 2025-2033.

8. Duggan A. Understanding interpersonal communication processes across health contexts: advances in the last decade and challenges for the next decade. J. Health Commun. 2006; 11 (1): 93-108.

9. Matthews AK, Sellergren SA, Manfredi C, et al. Factors influencing medical information seeking among African American cancer patients. J. Health Commun. 2002; 7 (3): 205-219.

10. Masami M, Takayuki S, Nguyen VD, et al. (2017), "Prevalence, perception and factors associated with diabetes mellitus among the adult population in central Vietnam: a population-based, cross-sectional seroepidemiological survey". BMC Public Health 17:298.

\title{
KHẢO SÁT ĐĂC ĐIỂM LÂM SÀNG KHẢ NĂNG PHỤC HỒI NGÔN NGŨ̃ CỦA TRẺ ĐIỂC BẨM SINH SAU PHẪU THUÂTT CẤY ỐC TAI ĐIỆN TỬ
}

\author{
Nguyễn Thanh Vũ*, Phạm Thị Minh Anh*, Đặng Thị Dượt*,
} Nguyễn Ngọc Thành Đạt*, Huỳnh Hoàng Minh*, Chiêm Hoàng Nhân*.

\section{TÓM TẮT}

Mục tiêu: Khảo sát các đặc điểm lâm sàng và sự phuc hồi thính giác của trẻ sau phấu thuât cây ốc tai điên tử. Thiết kế nghiên cứu: Hồi cứu và tiến cứu mồ tả loạt ca. Phương pháp: Khảo sát các đặc điểm lâm sàng và khả năng phục hồi thính giác của trẻ qua đánh giá lâm sàng trên thang điểm CAP. Kết quả: Chúng tôi nghiên cứu 39 trẻ điếc bẩm sinh đã phẫu thuât cấy ốc tai điện tữ tai Bênh viên Tai - Mũi - Họng TPHCM từ 01/2018 đến 04/2020 gồm 20 trẻ nam và 19 trẻ nữ. Đố tuổi phâ̂u thuật từ 1 đến 13 tuổi (trung bình $3.92 \pm 2.87$ tuổi), trong đó đa số trẻ cấy ốc tai điên tử tữ 1 đến 3 tuối $(61.54 \%)$. Số trẻ được cấy môt tai là $23 / 39$ trẻ $(58,97 \%)$. Số trẻ được cấy hai tai là $16 / 39$ trẻ $(41,03 \%)$. Sau phẫu thuật 1 năm, tất cả trẻ đat CAP từ 5 điểm trở lên, trong đó $87.18 \%$ trẻ đat CÁP từ 6 đến 7 điểm. CAP trung bình sau 1 năm cẩy

*Đại học Quốc Gia - Tp. Hồ Chí Minh

Chiu trách nhiệm chính: Nguyễn Thanh Vũ

Email: bsntvu@gmail.com

Ngày nhận bài: 18.10.2021

Ngày phản biện khoa học: 20.12 .2021

Ngày duyệt bài: 29.12.2021 ốc tai điện tử là $6.18 \pm 0.64$. Kết Iuận: Chúng tôi nghiên cứu 39 trẻ điếc bẩm sinh gồm 20 trẻ nam và 19 trẻ nữ. Độ tuổi phẫu thuật từ 1 đến 13 tuổi). Số trẻ được cây một tai là $23 / 39$ trẻ $(58,97 \%)$. Điểm CAP của tất cả trẻ có xu hướng tăng mạnh và về gần bình thường trong vòng 1 năm sau phấu thuật. Cấy ốc tai điện tử 2 bên cho hiệu quả phục hồi thính giác cao hơn.

Tư khóa: nghe kém, điếc bẩm sinh, ốc tai điện tử, phục hồi thính giác.

\section{SUMMARY}

\section{DETERMINE THE CLINICAL FEATURES AND EFFECTS OF AUDITORY REHABILITATION IN CHILDREN AFTER OF COCHLEAR IMPLANTATION}

Purpose: This study aimed to determine the clinical features and effects of auditory rehabilitation in children after of cochlear implantation. Study design: Retrospective and prospective descriptive study. Method: Determine the clinical features and effect $s$ of auditory rehabilitation in children according to the CAP scale. Result: Thirty nine children with congenital deafness (20 boys and 19 girls) who received cochlear implants at the Ho Chi Minh City Otorhinolaryngology Hospital (January 2018-April 2020) were included in this study. Age at CI ranged from 1 to 13 years (mean 
age was $3.92 \pm 2.87$ years) and most underwent $\mathrm{CI}$ at the age of 3 years or less (61.54\%). Number of children with cochlear implants one ear is 23/29 children $(58.97 \%)$. The number of children with bilateral CI are 16/29 children (41.03\%). At 1 year after $\mathrm{CI}$, all children reached the CAP score at 5 points or more and $87.18 \%$ of cases had CAP score from 6 to 7 points. The mean CAP scores at 12 months after CI were $6.18 \pm 0.64$ points. Conclusion: Thirty nine children with congenital deafness (20 boys and 19 girls) who received cochlear implants were included in this study. Age at CI ranged from 1 to 13 years and most underwent $\mathrm{CI}$ at the age of 3 years or less $(61.54 \%)$. Number of children with cochlear implants one ear is $23 / 29$ children $(58.97 \%)$. The CAP scores of all children tended to increase sharply and return to near normal within 1 year after CI. Children with bilateral $\mathrm{CI}$ had better auditory performance than children with unilateral CI.

Keywords: hearing loss, congenital deafness, cochlear implantation (CI), auditory rehabilitation.

\section{I. ĐĂTT VẤN ĐỀ}

Điếc câm bẩm sinh là khiếm khuyết về giác quan thường gặp nhất, gây ảnh hưởng nghiêm trọng đến sinh hoạt xã hội, sự phát triển tâm sinh lý của bệnh nhân, đặc biệt là ảnh hưởng rất nhiều đến tương lai của trẻ em. Theo Tổ chức $Y$ tế thế giới (WHO) có 430 triệu người nghe kém (chiếm $5 \%$ dân số), trong đó có 34 triệu trẻ em. Trong đó, khu vực Châu Á Thái Bình Dương đứng thứ 2 về tỉ lệ nghe kém ở trẻ em $(2 \%)$, sau Nam Á (2.4\%).

Tại Việt Nam, theo Bộ Lao động Thương binh và Xã hội, đến năm 2003 có 662 ngàn trẻ bị khuyết tật (2.4\%). Trong đó, rối loạn thần kinh và khiếm thính phổ biến thứ hai (17\%), sau khuyết tật vận động (29\%)[1]. Theo báo cáo của Người khuyết tật năm 2009 có khoảng 3 triệu người khiếm thính (chiếm $3.8 \%$ dân số), trong đó trẻ em chiếm $0.5 \%$ với tỉ lệ nam nhiều hơn nữ [2].

Phẫu thuật cấy ốc tai điện tử bắt đầu nhờ vào sự phát triển của các thiết bị ốc tai điện tử đơn kểnh vào những năm 60 của thế kỉ trước. Mười năm sau đó, các thiết bị ốc tai đa kênh phát triển cùng sự xuất hiện ngày càng nhiệu của những trung tâm cấy ổc tai điện tử ở Úc, Mỹ, Pháp, Thụy sĩ. Ngày 1/8/1978, Graeme Clark đã cấy ghép thiết bị ốc tai điện tử đa kệnh đầu tiên trên thế giới cho một bệnh nhân tại Úc.

Tại Việt Nam, BV Tai Mũi Họng TP.HCM thực hiện ca phẫu thuật đầu tiên vào năm 1998. Đến nay có hơn 500 trường hợp thành công, giúp bệnh nhân phát triển khả năng nghe, nói, học tập và hòa nhập với xã hội.

Nhận thấy điếc câm bẩm sinh sẽ ảnh hưởng rất nhiều đến sự phất triển toàn diện của trẻ và phẫu thuật cấy ốc tai để phục hồi ngôn ngữ sẽ mang lại lợi ích lớn và thực sự cần thiết. Việc phát hiện sớm và can thiệp kịp thời sẽ mang lại cho trẻ cơ hội lớn trong việc hồi phục khả năng nghe, phát triển các kỹ năng ngôn ngữ, giúp trẻ học tập, hòa nhập cộng đồng và giảm gánh nặng cho bản thân trè, gia đình và xã hội [2]. Do đó, chúng tôi tiến hành thực hiện đề tài nghiên cứu này, nhằm mục đích nhìn nhận rõ về các đặc điểm lâm sàng và khả năng phục hồi ngôn ngữ sau phẫu thuật, đồng thời đưa ra các mốc tham khảo về sự phục hồi tại các thời điểm sau cấy. Mục tiêu tổng quát: Khảo sát các đặc điểm lâm sàng và mức độ phục hồi thính giác của trẻ theo thang điểm CAP sau cấy ốc tai điện tử. Mục tiêu cụ thể:

1. Khảo sát các đặc điểm lâm sàng của trẻ điếc câm bẩm sinh.

2. Xác định mức độ phục hồi thính giác sau cấy 1 năm: điểm CAP tối thiểu và tỉ lề trẻ đat CAP tối đa; xu hướng phục hồi thính giác; điểm CAP trung binh tại mốc 3, 6, 9 và 12 tháng.

\section{II. ĐỐI TƯƠ'NG VÀ PHƯƠNG PHÁP NGHIÊN CỨU}

Đối tượng nghiên cứu: Trẻ điếc bẩm sinh đã được phẫu thuật cây ốc tai điện tử từ tháng 01/2018 đến tháng 04/2020 tại Bệnh viện Tai Mũi Họng TP. Hồ Chí Minh.

Phương pháp nghiên cứu: Nghiên cứu mô tả loạt ca.

Kî̀ thuật chọn mẫu: Mẫu toàn bộ: 39 trẻ thỏa tiêu chuẩn chọn mẫu.

Phương pháp thu thập và xử lý số liệu: Chúng tôi thu thập số liệu bằng bảng câu hỏi và xử lý số liệu bằng phần mềm Stata 16.0.

Phương pháp hạn chế sai số: rõ ràng.

- Thiết kế bộ phiếu thu thập đúng mục tiêu,

- Tuân thủ nghiêm ngặt tiêu chí đưa vào và loai ra.

- Định nghĩa rõ ràng các thông tin được thu thập trong nghiên cứu.

- Đảm bảo thu thập được đủ các thông tin cần thiết cho nghiên cứu.

Phương pháp đánh giá: chúng tôi khảo sát các đặc điểm lâm sàng và đánh giá khả năng phục hồi ngôn ngữ của trẻ bằng thang điểm CAP (Categories of Auditory Performance), gồm 8 mức độ:

0: Không quan tâm đến âm thanh môi trường.

1: Có quan tâm đến âm thanh môi trường.

2: Có đáp ứng với tiếng nói.

3: Nhận biết được âm thanh môi trường.

4: Phần biệt được ít nhất 2 từ.

5: Hiểu những cụm từ đơn giản không nhìn miệng. 
6: Hiểu cuộc nói chuyện với người thân không nhìn miệng.

7: Có thể dùng điện thoại với người thân.

\section{KẾT QUẢ NGHIÊN CỨU}

Chúng tôi nghiên cứu 39 trẻ đã được phẫu thuật cấy ốc tai điện tử, ghi nhận các đặc điểm sau:
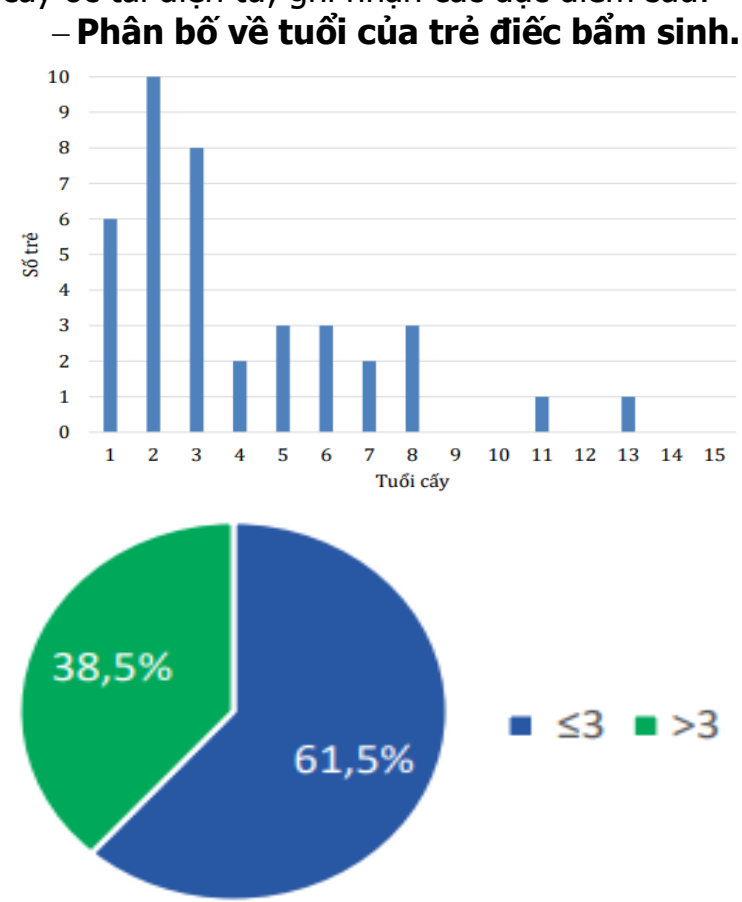

Biểu đồ 1. Tuổi cây ốc tai và độ tuổi cây nhiều nhất
Nhận xét: Tuổi cấy ốc tai trong nghiên cứu này từ 1 đến 13 tuổi (tuổi trung bình là 3.92), độ tuổi trẻ được cấy nhiều nhất là từ 1 đến 3 tuổi.

- Phân bố về giới của trẻ điếc bẩm sinh.

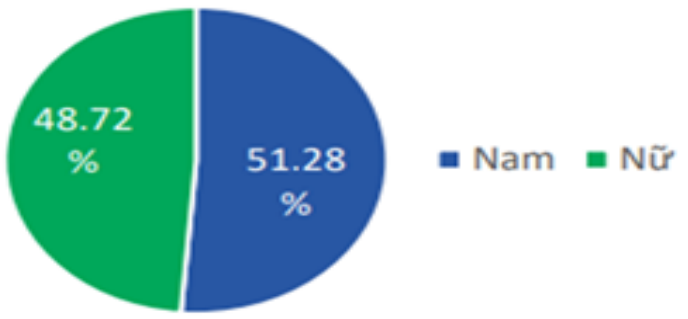

Biểu đồ 2. Phân bố giới tính

Nhận xét: Nam chiếm 20/39 trẻ (51.28\%) và nữ chiếm 19/39 trẻ (48.72\%). Tỉ lệ nam/nữ là 1.05.

-Số tai được cấy ở trẻ điếc bẩm sinh.

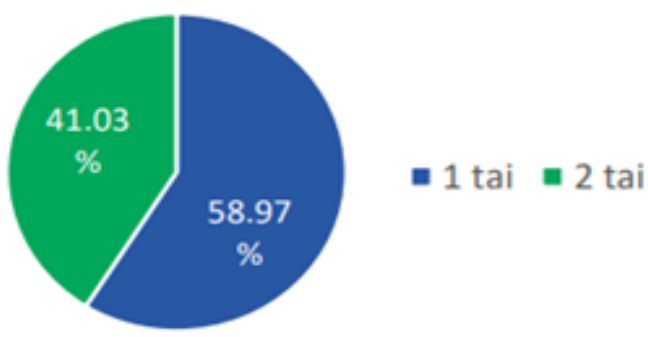

Biểu đồ 3. Phân bố về số tai cây

Nhận xét: Số trẻ được cây một tai là 23/39 trẻ $(58,97 \%)$. Số trẻ được cây hai tai là $16 / 39$ trẻ $(41,03 \%)$

- Đặc điểm về thính học ở trẻ điếc bẩm sinh.

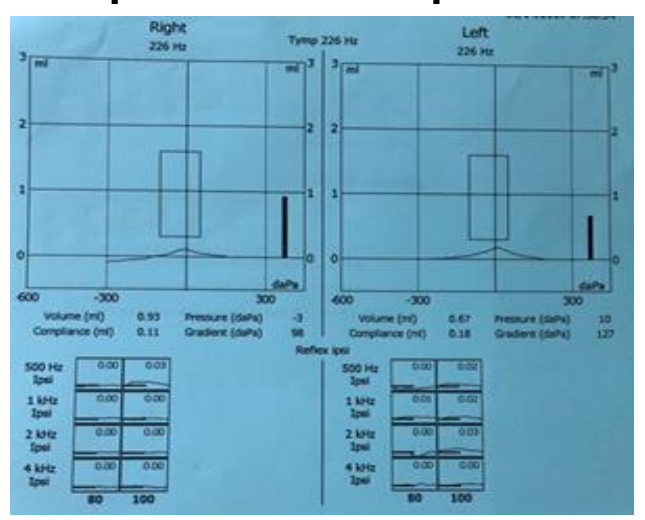

Nhĩ lượng đồ và phản xạ cơ bàn đạp

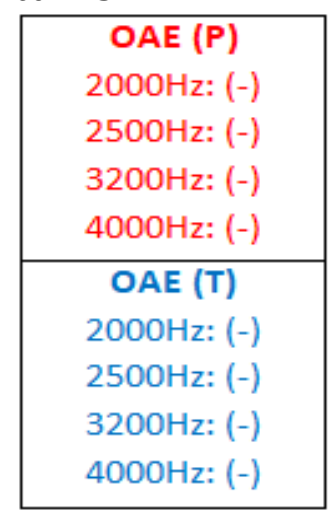

OAE

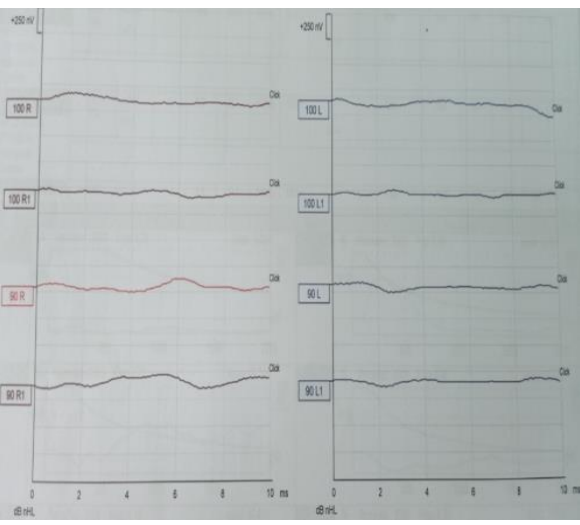

$\boldsymbol{A B R}$

Biểu đồ 4. Các đăc điểm thính hoc của trẻ điếc bẩm sinh

Nhận xét: Tất cả trẻ có chỉ định phẩu thuật đều được chẩn đoán điếc sâu (>90db): đo nhĩ lượng đồ dạng $A$ và phản xạ cơ bàn đạp âm tính. Được kiểm tra thính lực chuyên sâu bằng âm cách đo ốc tai (OAE: không đáp ứng các tầng số) và điện thính giác thân não (ABR: không xuất hiện sóng $V$ ở cường độ 112dB).

-Xu hướng phục hồi thính giác của trẻ. 


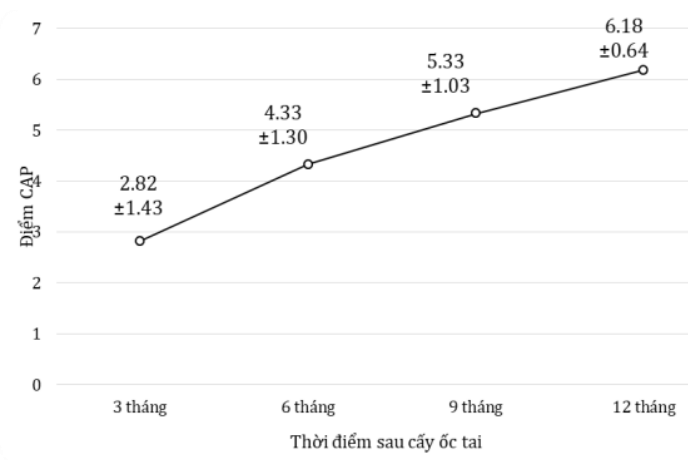

Biểu đồ 5. Xu hướng phục hồi thính giác của trẻ theo điểm CAP trung binh.

Nhân xét: Điểm CAP trung bình của trẻ đạt được ở các mốc thời điểm 3 tháng, 6 tháng, 9 tháng, 12 tháng sau cấy ốc tai điện tử là 2.82, $4.33,5.33,6.18$. Kết quả này được biểu diễn bằng một đường thẳng đi lên chứng tỏ rằng mức độ cải thiện khả năng nghe của trẻ luôn tăng dần theo thời gian. Sau một năm, điểm CAP trung bình của trẻ đạt được gần như tối đa (6.18 $\pm 0.64)$.

- Mức độ phục hồi thính giác của trẻ tại mốc phân cách 3 tuổi sau cây 12 tháng.

Bảng 2. Điểm CAP sau cây ốc tai 12 tháng theo nhóm tuổi.

\begin{tabular}{|c|c|c|c|c|c|c|c|c|c|}
\hline $\begin{array}{c}\text { Nhóm } \\
\text { tuối }\end{array}$ & 0 & 1 & 2 & 3 & 4 & 5 & 6 & 7 & \multirow{2}{*}{$\begin{array}{c}\text { Trung } \\
\text { bình }\end{array}$} \\
\hline$\leq 3$ & - & - & - & - & - & $5(20.83 \%)$ & $13(54.17 \%)$ & $6(25.00 \%)$ & $6.04 \pm 0.69$ \\
\hline$>3$ & - & - & - & - & - & - & $9(60.00 \%)$ & $6(40.00 \%)$ & $6.40 \pm 0.51$ \\
\hline
\end{tabular}

Nhận xét: Ớ nhóm $\leq 3$ tuổi, tất cả trẻ đạt CAP từ 5 điểm trở lên(CAP trung bình là 6.04 \pm 0.69 ), trong đó $20.83 \%$ trẻ đạt CAP 5 điểm, 54.17\% trẻ đạt CAP 6 điểm, 25\% trẻ đạt CAP 7 điểm. Nhóm $>3$ tuổi, tất cả trẻ đạt CAP từ 6 điểm trở lên (CAP trung bình là $6.40 \pm 0.51$ ), trong đó $60 \%$ trẻ đạt CAP 6 điểm và $40 \%$ trẻ đạt CAP 7 điểm. Như vậy, sau cấy ốc tai 12 tháng, cả hai nhóm tuổi đều có CAP đạt mức gần như phục hồi về bình thường.

- Mức độ phục hồi thính giác của trẻ theo số tai phẫu thuật

Bảng 3. Điểm CAP trung binh theo số tai cây.

\begin{tabular}{|c|c|c|c|c|}
\hline \multirow{2}{*}{ Số tai cấy } & \multicolumn{4}{|c|}{ Điểm CAP trung bình } \\
\cline { 2 - 5 } & 3 tháng & 6 tháng & 9 tháng & 12 tháng \\
\hline Một tai & $2.91 \pm 1.65$ & $4.26 \pm 1.29$ & $5.22 \pm 1.00$ & $6.04 \pm 0.64$ \\
\hline Hai tai & $2.68 \pm 1.08$ & $4.44 \pm 1.36$ & $5.50 \pm 1.10$ & $6.38 \pm 0.62$ \\
\hline
\end{tabular}

Nhận xét: Đối với nhóm trẻ được cấy một tai, CAP trung bình đạt được ở 3 tháng đầu là 2.91, đến tháng thứ 12 tăng lên 6.04 điểm. Đối với nhóm trẻ được cấy hai tai, CAP trung bình đạt được ở 3 tháng đầu là 2.68 điểm, đến tháng thứ 12 tăng lên 6.38 điểm

\section{BÀN LUÂN}

Trong nghiên cứu này, chúng tôi đánh giá các đặc điểm lâm sàng và sự phục hồi ngôn ngữ của trẻ bằng thang điểm CAP có 8 mức độ từ 0-7. Tất cả trẻ có chỉ định phẩu thuật đều được chẩn đoán điếc sâu (>90db), tương đương CAP nhận vào bằng 0 điểm. Chúng tôi đưa ra những bàn luận sau:

- Về đặc điểm lâm sàng: Tất cả trẻ có chỉ định phẩu thuật đều được chẩn đoán điếc sâu (>90db), có nhĩ lượng đồ dạng $A$ và phản xạ cơ bàn đạp âm tính. Được kiểm tra thính lực chuyên sâu bằng âm cách đo ốc tai (OAE) và điện thính giác thân não (ABR).

- Hiệu quả phục hồi thính giác sau cây: Theo số liệu nghiên cứu của chúng tôi, tất cả trẻ được cấy ốc tai điệu tử đều có cải thiện khả năng nghe - nói ở các mức độ khác nhau. Điểm CAP trung bình của trẻ cải thiện rõ, xu hướng luôn tăng dần theo thời gian. So sánh với nghiên cứu của Min Young Kwak (2020) thực hiện trên
114 trẻ được cấy ốc tai điện tử có độ tuổi từ 1 13 ghi nhận điêm CAP cũng cải thiện sau phẫu thuật. Cụ thể, các mốc thời điểm 3 tháng, 6 tháng, 12 tháng là $2.30-4.60 ; 3.40-5.30 ; 4.70$ - 5.50[8]. Nghiên cứu của Hsuan-Yeh Fang (2014) thực hiện trên 84 trẻ nhỏ hơn 5 tuổi được cấy ốc tai điện tử cho ra kết quả điểm CAP ở thời điểm 6 tháng và 12 tháng sau cấy là 2.00 và 4.00 [9]. Như vậy có sự tương đồng về khả năng phục hồi ngôn ngữ giữa nghiên cứu của chúng tôi so với các nghiên cứu khác trên thế giới. Tất cả trẻ được cây ốc tai điện tử đều cải thiện điểm CAP theo thời gian. Tuy nhiên, kết quả nghiên cứu của chúng tôi cho thấy rằng mức độ cải thiện của trẻ có phần vượt trội hơn so với các nghiên cứu khác. Để lý giải cho điêu này, chúng tôi đưa ra 2 lý do: thứ nhất, về thời điểm thực hiện nghiên cứu, nghiên cứu của Hsuan-Yeh Fang thực hiện vào năm 2014, của Bakhshaee thực hiện vào năm 2007, trong khi nghiên cứu của chúng tôi được hiện vào năm 2021, sau gần 10 năm, các phương pháp phẫu thuật ngày càng 
cải tiến và hồi phuc tốt hơn, chất lượng thiết bi ốc tai điện tử và chương trình hồi phục ngôn ngữ ngày càng được cải thiện hơn, đồng thời có sự quan tâm nhiều hơn của gia đình, xã hội đối với trẻ. Thứ hai, đó là do sự khác biệt về đặc điểm xã hội, văn hóa ở từng quốc gia thực hiện nghiên cứu, cha me và gia đình có áp lực công việc và cuộc sống khác nhau, nên mức độ quan tâm đối với trẻ không giống nhau, điều này sẽ ảnh hưởng đến hiệu quả hồi phục ngôn ngữ của trẻ.

- Tuổi cấy ốc tai: Theo số liệu thống kê, sau 12 tháng cấy ốc tai điện tử, tất cả các trẻ ở 2 nhóm tuổi đều có sự cải thiện mức độ thính giác đáng kể với CAP đạt từ 5 điểm trở lên. Điểm CAP trung bình sau 12 tháng cấy ốc tai điện tử của nhóm trẻ dưới 3 tuổi là $6.04 \pm 0.69$ điểm và nhóm trẻ trên 3 tuổi là $6.40 \pm 0.51$ điểm. Đánh giá cụ thể nhóm trẻ dưới 3 tuổi, CAP sau 12 tháng đạt từ 6 điểm trở lên chiếm tỷ lê gần $80 \%$. Ở độ tuổi này, trẻ khó đạt CAP 7 điểm (vì chưa thể sử dụng điện thoại với người thân), đa số CAP 6 điểm đã phù hợp với lứa tuổi (trẻ hiểu cuộc nói chuyện với người thân mà không nhìn miệng). Ngược lại, nhóm trẻ trên 3 thì khả năng đạt điểm CAP tối đa cao hơn, tuy nhiên chưa tới $50 \%$ trẻ đạt CAP 7 điểm. Điều này cho thấy cây ở lứa tuổi sớm hơn sẽ đạt kết quả phục hồi ngôn ngữ tốt hơn, CAP cải thiện phù hợp với tuổi đáng kể. Nghiên cứu của Dettman (2016) trên 403 trẻ cho kết luận những trẻ dưới 2 tuổi cho hiệu quả phục hồi ngôn ngữ cao hơn nhóm trẻ còn lại được đánh giá theo thang điểm CNC test [7]. Tương tự kết quả nghiên cứu của Shaofeng Liu (2019) trẻ được cây dưới 7 tuổi cho hiêuu quả phục hồi tốt hơn theo dõi trong 10 nắm bởi thang điểm CAP và ASK-WRSs [4], nghiên cứu Quianquaian (2020) cây sớm sẽ mang lại lợi ích tốt hơn cho sự phát triển ngôn ngữ của trẻ được theo dõi trong 5 năm theo thang điểm CAP và SIR [6]. Như vậy, các nghiên cứu đều chỉ ra rằng trẻ điếc bẩm sinh được cấy ốc tai sớm sẽ mang lại lợi ích phát triển ngôn ngữ tốt hơn. Điều này cũng tương đồng với nghiên cứu của chúng tôi, nhóm tuổi cấy nhỏ hơn 3 đạt khả năng phục hồi ngôn ngữ phù hợp với lứa tuổi tốt hơn so với nhóm tuổi lớn hơn 3.

-Số tai phẫu thuật: sau cãy ốc tai 12 tháng, cả 2 nhóm trẻ cẩy một tai và hai tai đều đạt CAP từ 5-7 điểm, đa số trẻ ở 2 nhóm đều đạt CAP 6 điểm: $60.87 \%$ đối với nhóm trẻ được cấy một tai và $50 \%$ đối với nhóm trẻ cây hai tai. CAP trung bình sau cấy 12 tháng ở nhóm cáy hai tai cao hơn nhóm cây một tai là 6.38 và 6.04 . Kết quả của chúng tôi tương đồng với nghiên cứu của Gökay [5] cũng có kết quả thính lực khi cấy hai bên cao hơn một bên tai. Uuu điểm của của cấy hai tai theo Gregory [3] là nghe lời nói trong môi trường ồn, cải thiện khả năng hội thoại và khả năng định vị âm thanh. Ngoài ra, Bradford đã sử dụng thang điểm đánh giá sự cải thiện chất lượng cuộc sống và thấy có sự cải thiện sau khi cấy tai thứ hai.

\section{KẾT LUÂ̂N}

Điểm CAP của tất cả trẻ có xu hướng tăng mạnh và về gần bình thường trong vòng 1 năm sau phẫu thuật. Cây ốc tai điện tử 2 bên cho hiệu quả phục hồi thính giác cao hơn. Số giờ học phục hồi ngôn ngữ và sự hợp tác của gia đình đóng vai trò quan trọng nâng cao hiệu quả phục hồi.

\section{TÀI LIÊU THAM KHẢO}

[1] Bộ Lao Động - Thương Binh - Xã Hội Và Unicef (2004), "Phân tích tình hình trẻ em khuyết tật ở Việt Nam", Nhà xuất bản Lao động Xã hội, Hà Nội.

[2] Quỹ̃ Dân Số Liên Hiệp Quốc-Unfpa Quỹ (2009), "Một số kết quả chủ yếu từ tổng điêu tra dân số và nhà ở Việt Nam năm 2009", Người khuyết tật Việt Nam, 16-17.

[3] G. J. Basura, R. Eapen\& C. A. Buchman (2009), "Bilateral cochlear implantation: current concepts, indications, and results", Laryngoscope, 119.(12) 2395-401.

[4] S. Liu, F. Wang, P. Chen, N. Zuo, C. Wu, J. Ma, J. Huang\& C. Wang (2019), "Assessment of outcomes of hearing and speech rehabilitation in children with cochlear implantation", J Otol, 14.(2) 57-62.

[5] N. Yıldırım Gökay\& E. Yücel (2021), "Bilateral cochlear implantation: an assessment of language sub-skills and phoneme recognition in school-aged children", Eur Arch Otorhinolaryngol, 278.(6) 2093-2100.

[6] Q. Guo, J. Lyu, Y. Kong, T. Xu, R. Dong, B. Qi, S. Wang\& $X$. Chen (2020), "The development of auditory performance and speech perception in CI children after long-period follow up", Am J Otolaryngol, 41.(4) 102466.

[7] S. J. Dettman, R. C. Dowell, D. Choo, W. Arnott, Y. Abrahams, A. Davis, D. Dornan, J. Leigh, G. Constantinescu, R. Cowan\& R. J. Briggs (2016), "Long-term Communication Outcomes for Children Receiving Cochlear Implants Younger Than 12 Months: A Multicenter Study", Otol Neurotol, 37.(2) e82-95.

[8] M. Y. Kwak, J. Y. Lee, Y. Kim, J. W. Seo, J. Y. Lee, W. S. Kang, J. H. Ahn, J. W. Chung\& H. J. Park (2020), "Long-term Change in the Speech Perception Ability in Pediatric Cochlear Implants and the Effect of the Age at Implantation", Otol Neurotol, 41.(6) 758-766.

[9] H. Y. Fang, H. C. Ko, N. M. Wang, T. J. Fang, W. C. Chao, Y. T. Tsou\& C. M. Wu (2014), "Auditory performance and speech intelligibility of Mandarin-speaking children implanted before age 5", Int J Pediatr Otorhinolaryngol, 78.(5) 799-803. 